\section{rev Psi}

Revista de Psicología (UNLP)

https://revistas.unlp.edu.ar/revpsi

\title{
Políticas públicas y producciones subjetivas. Accesibilidad y derecho a la salud
}

\author{
Agustina D'Agostino' Julieta Veloz'
}

Correspondencia

dagostinoag@gmail.com

Filiaciones institucionales

${ }^{1}$ Facultad de Psicología, Universidad Nacional de

La Plata (UNLP, Argentina)

\section{Resumen}

En este trabajo se problematiza el concepto de accesibilidad al derecho a la salud a partir de entender que las políticas sociales son el resultado de una construcción social compleja, que componen una red heterogénea de elementos discursivos y no discursivos que involucran relaciones de poder-saber y líneas de enunciación, de visibilización, de objetivación y de subjetivación. Se reflexiona acerca de la importancia de focalizar en los modos de producción de subjetividad y se interrogan los dispositivos que implementan los efectores de políticas públicas, de organización comunitaria, de transversalidad y de autonomía que ellos producen. Esto tiene consecuencias éticopolíticas en las acciones metodológicas de investigación acción. Esta última debe asumir un carácter local, situado y participativo. Cobra importancia el trabajo de intervención, el análisis de los imaginarios sociales y la capacidad de los dispositivos de promover agenciamientos de resistencia a las lógicas neoliberales.

\section{Palabras clave}

salud pública | producción de subjetividad | dispositivo | accesibilidad

\section{Cómo citar}

D'Agostino, A. y Veloz, J. (2021). Políticas públicas y producciones subjetivas. Accesibilidad y derecho a la salud. Revista de Psicología. HTTPs://DX.DOI. ORG/10.24215/2422572XE079

\section{Proceso editorial}

Recibido

8 may. 2020

Aceptado

3 feb. 2021

Editor

Nicolás Alessandroni | Facultad de Psicología, Universidad Autónoma de Madrid (España)
ISSN

2422-572X

Licencia

Licencia de Cultura Libre CC-BY 4.0

(Compartir - Adaptar - Atribuir)

Entidad editora

RevPsi es una publicación de la

Facultad de Psicología (Universidad

Nacional de La Plata, Argentina) 


\section{Políticas públicas e produções subjectivas. Acessibilidade e 0 direito à saúde}

\section{Resumo}

Neste artigo problematiza-se o conceito de acessibilidade ao direito à saúde, partindo do entendimento que as políticas sociais são o resultado de uma construção social complexa, componentes de uma rede heterogênea de elementos discursivos e não discursivos, que envolvem relações de poder- saber, linhas de enunciação e de visualização, de objetivação e subjetivação. É preciso refletir sobre a importância de focalizar os modos de produção da subjetividade e pesquisar os dispositivos que implementam os efeitores de políticas públicas e os modos de construção do comum, da organização comunitária e da transversalidade e autonomia que eles mesmos produzem. Isto tem consequências éticas e políticas nas ações metodológicas da investigação ação, a qual deve assumir um caráter local, situado e participativo. Obtém relevância o trabalho da intervenção, do análise dos imaginários sociais e a capacidade dos dispositivos para promover agenciamentos de resistência as lógicas neoliberais.

\section{Palavras-chave}

saúde pública | produção de subjetividade | dispositivo | acessibilidade

\section{Public policies and subjective productions. Accessibility and the health right}

\section{Abstract}

This article problematizes the concept of accessibility to the right to health, based on the idea that public policies are the result of a complex social construction, components of a heterogeneous ensemble consisting of discursive and non-discursive elements, and involving knowledge - power relations and lines of visibility and enunciation, objectification and subjectification. A reflection is made upon the importance of focusing on the ways in which subjectivity is produced, and questions are raised about the apparatuses employed by public policy makers and about the modes of construction of commonness, community organization, intersectionality and autonomy that these produce. This has ethical and political implications for action methodological actions, which must assume a local, situated and participatory character. Importance is therefore attributed to social intervention work, the analysis of social imaginaries, and apparatuses' ability to promote agencies of resistance against neoliberal logics.

\section{Keywords}

public health | production of subjectivity | apparatus (dispositif) | accessibility 


\section{Aspectos destacados del trabajo}

- La noción de dispositivo aporta a las políticas públicas el análisis de relaciones de saber-poder.

- Los Estados liberales producen políticas sociales en lógica de servicio y modos de subjetivación usuarios/consumidores.

- Los dispositivos institucionales pueden producir subjetividades para el ejercicio de los derechos y producir salud.

- Las intervenciones situadas transforman los focos locales poder y resistencia.

El presente escrito parte de la sistematización de las prácticas acontecidas desde un proyecto de extensión en un barrio en la ciudad de La Plata, a la luz de reflexionar acerca del ejercicio al derecho a la salud. Es decir, que se trata de un trabajo teórico de reflexión sobre una práctica en territorio, llevada a cabo por extensionistas, investigadores, docentes, trabajadores de la salud.

Nuestro acercamiento a la problemática partió de un pedido realizado por una referente territorial a partir de diferentes situaciones de violencias que se presentaban en el barrio y de la ausencia de dispositivos de atención en salud y en salud mental en territorio.

Como primer paso nos propusimos indagar la accesibilidad a los servicios de salud, a partir de un mapeo y la realización de entrevistas abiertas e informales a referentes y vecinos. A partir de este primer acercamiento, se presentaron diferentes cuestionamientos que nos condujeron a reflexionar sobre los conceptos de los que partíamos y a reformular redes teóricas y modos de abordaje.

En un primer apartado presentamos la noción de accesibilidad, ya que nuestro propósito como equipo consistía en realizar un relevamiento de los obstáculos y facilitadores presentados y los elementos relevados a partir del mapeo en territorio. A partir de allí, reflexionamos acerca de los alcances y limitaciones que entendemos presentó para nosotros esta noción a partir de la construcción de nuevas preguntas de investigación: ¿Acceder a un servicio implica el ejercicio de un derecho? ¿Qué tipo de subjetividades producen los dispositivos que se implementan en territorio?

A partir de estos interrogantes, tomamos diferentes desarrollos teóricos vinculados a los aportes e intervenciones de los psicólogos para la producción de subjetividad en el campo de las políticas públicas (Calmels, 2015; del Cueto, 2014; D’Agostino, Vidal y Veloz, 2018; Fernández, 2011; Pérez 2014; Stolkiner, 2015; Ulloa, 2011).

Para finalizar, presentamos la experiencia realizada y realizamos una breve reflexión 
sobre los posibles aportes a las políticas públicas en marco de derechos a partir de incorporar intervenciones que se proponen producir subjetividad. Cómo equipo de trabajo, nos ocupan especialmente las problemáticas que se presentan en la comunidad. Construimos desde allí interrogantes y lecturas acerca de la integración de los ámbitos públicos, y los modos en que se vincula la Universidad pública, el conocimiento que produce, los programas de formación que brinda y el aporte que desde estas experiencias hacia las políticas públicas. Tensionar cuáles son las dimensiones políticas de la investigación, la extensión y la decencia en la Universidad (Fernández, 2008; Pérez, 2018; Stolkiner, 2008).

\section{¿Qué entendemos por políticas públicas? Una primera aproximación}

Partimos de concebir que los procesos generadores y estructuradores de políticas sociales incluyen actores con intereses, ideas y representaciones sociales varias y que, a su vez, los modelos de política pública que se formulan integran lógicas diversas entre asistencia y protección social, cohesión y control social, derechos sociales y constitución de ciudadanía (Fleury, 2002; Danani, 2012).

Aguilo (2005) sostiene que la existencia o no de lo que se llama políticas sociales está profundamente determinada por el tipo de Estado presente en la sociedad y por el modelo de crecimiento adoptado en esa sociedad, pero, además, señala que estas concepciones introducen las categorías de población, de condiciones de vida y de acción del Estado. El autor caracteriza las políticas sociales como: (a) la resultante de una acción estatal, característica que comparte con las políticas públicas; (b) acciones que se traducen en la regulación, provisión o disposición por parte del estado de ciertos bienes y/o servicios; (c) bienes y/o servicios que se pueden disponer para toda la sociedad, para ciertos grupos caracterizados previamente o para ciertos individuos calificados por antelación. La definición de los receptores de estas acciones estatales se relaciona con (d) los objetivos de las políticas sociales, que componen el cuarto elemento que las caracteriza.

Cuando las políticas de gobierno se inscriben en un enfoque de derechos e inclusión social ello implica el nacimiento de las obligaciones positivas del gobierno traducido en políticas públicas. Así, los derechos humanos se vuelven guía ordenadora de las obligaciones de los gobiernos y del alcance de la participación social en los mecanismos de control y responsabilidad tanto a nivel local como internacional. Al reconocer a los sectores excluidos como titulares de derechos que obligan al Estado, se modifica la lógica de políticas elaboradas para la asistencia de personas con necesidades por la de sujetos con derecho a demandar prestaciones y conductas. Desde esta perspectiva, cobran importancia la participación, la información y la accesibilidad (Abramovich, 2006; Pautassi, 2010). 


\section{Sobre la accesibilidad}

La accesibilidad de la población al sistema de salud es un elemento fundamental de la Atención Primaria de la Salud (APS), definida en la Declaración de Alma-Ata de 1978 y reafirmada por la Organización Panamericana de la Salud (OPS) en el 2007.

Ha sido precisada tradicionalmente como la forma en que los servicios se acercan a la población, una cuestión de la oferta que implica eliminar diferentes barreras: geográficas, económicas, administrativas y culturales (Carrizo Villalobos, 2016).

De acuerdo con lo planteado por Comes y Stolkiner (2004) se han presentado diferentes clasificaciones según el tipo de obstáculos o facilitadores que se presentan a la accesibilidad:

- La accesibilidad económica refiere a la posibilidad de acceso por cuestiones monetarias, podríamos pensarlo a partir de la existencia y cantidad de servicios públicos de salud en el territorio, la cantidad de población sin cobertura o con cobertura según prestaciones, la cantidad de población con pensión económica asignada, la existencia de programas de provisión gratuita de medicamentos y el porcentaje de la población que consulta en los servicios de salud, entre otros aspectos.

- La accesibilidad administrativa se basa en la organización de los servicios, aquí podríamos analizar entre otros aspectos, el porcentaje de profesionales, técnicos y variedad de disciplinas en territorio de acuerdo con la cantidad de población, la existencia de derivaciones y articulaciones entre distintos servicios públicos, la cantidad de turnos disponibles por semana en los centros según la cantidad de población que asiste, la existencia de mecanismos de toma de turno no personales o electrónicos y la amplitud del horario de atención de los servicios.

- La accesibilidad geográfica se observa a partir de cuestiones espaciales o arquitectónicas. Por ejemplo, la existencia de caminos transitables entre los centros de salud y las viviendas, la disponibilidad de transporte público que conecte el centro de salud con los puntos habitacionales de usuarios, la cantidad de servicios de salud cercanos al domicilio, la disponibilidad de transportes de acuerdo con la población que requiere traslado entre los servicios de salud, la existencia de programas de atención comunitaria, los accesos para movilidad reducida, el porcentaje de población a más de 1 hora de un centro de salud, la existencia de dispositivos territoriales, la existencia de programas públicos de atención y promoción de la salud, la posibilidad de derivación a otros servicios y cercanía con los mismos y la articulación de los servicios con otros niveles de atención (municipales, programas nacionales). 
- Accesibilidad simbólica o cultural: se sustenta en considerar las variaciones culturales, de representaciones y sentidos entre el personal de los servicios y la población. Podemos incluir aquí el análisis de los sentidos, prácticas y afectos acerca de la salud y la enfermedad de los efectores y usuarios de la salud, existencia de campañas de promoción de la salud que tengan en cuenta el lenguaje y los medios de difusión al que recurra la comunidad, existencia de programas de promotoras de salud mental en el territorio, conocimiento del contenido del consentimiento informado por parte de efectores y usuarios/as, como elementos a considerar, entre otros.

Entendemos que, si bien la división planteada es arbitraria y parcial, constituye un aporte en tanto permite observar y analizar diversos aspectos que permiten o son obstáculos al momento del acceso a servicios, variable significativa para el ejercicio del derecho.

No obstante, nos encontramos con algunas observaciones respecto al concepto de accesibilidad. Landini, González Cowes y D’Amore (2014) analizan los modos en los que se ha presentado la noción de accesibilidad en diferentes artículos e investigaciones y plantean que muchas de estas nociones encuentran sus limitaciones al invisibilizar el impacto de la atención en salud. Proponen entonces la accesibilidad psico-sociocultural recurriendo al concepto de interfaz social en tanto "espacio de actualización de discontinuidades sociales en el que se encuentran actores que poseen diferentes racionalidades culturales o que forman parte de grupos sociales diferentes". Este concepto amplía el universo de actores: planificadores, autoridades institucionales, profesionales de la salud y comunidad, todos ellos tienen intereses, representaciones y referencias diversas. Se pone el énfasis en las rupturas, discontinuidades, conflictos y el contexto en el que se desenvuelven, lo que implica el análisis de los procesos de negociación y conflicto además de la consideración de las variaciones culturales.

Comes y Stolkiner (2005), desde una perspectiva relacional, sostienen que la accesibilidad consiste en el vínculo que se establece entre los servicios y los sujetos destinatarios de estos, a partir de concebir a ambas partes como portadoras de la posibilidad o imposibilidad de encontrarse. Solitario, Garbus y Stolkiner (2008), proponen que la accesibilidad sea entendida como un vínculo entre los sujetos y los servicios que surge de la combinación entre las condiciones y discursos de los servicios y las condiciones y representaciones de los/as usuarios/as, es decir, una condición simbólica. En este mismo trabajo, las autoras establecen una interesante vinculación entre el ejercicio de los derechos y la participación: los/as usuarios/ as sin participación en salud muestran mayores personalismos, tanto para el agradecimiento como para los reclamos, que se vinculan a la noción de "derecho selectivo" no universal. Desde la perspectiva de la accesibilidad simbólica se plantea que las distintas interpretaciones del derecho a la atención de la salud generan posiciones subjetivas disímiles con relación a la utilización de servicios, que impactan en el ejercicio del derecho (Comes, Solitario, Garbus, Mauro, Czerniecki y Vázquez, 
2007). La accesibilidad simbólica o cultural, incluye las variaciones culturales, de representaciones y sentidos, entre el personal de los servicios y la población (Carrizo Villalobos, 2016; Comes \& Stolkiner, 2007; Solitario, Garbus, Stolkiner, 2008). Pensar la accesibilidad en términos relacionales y tener en cuenta las dimensiones culturales y simbólicas permite analizar mejor la complejidad del fenómeno: tanto el imaginario social como las representaciones sociales, los sentidos y los afectos que orientan las prácticas pueden constituirse en barreras, obstáculos o facilitadores para el acceso a la salud.

Queda en evidencia que no sólo debemos indagar la posibilidad institucional de ser atendido desde una institución pública que se encargue de un área determinada a la que se acceda, sino que es necesario atender a ejercer el derecho a la salud (Domínguez Lostaló, 1996).

\section{Una segunda aproximación. Políticas públicas, dispositivos y producción de subjetividad}

A partir de entender a las políticas sociales como el resultado de una construcción social compleja, donde los espacios de negociación políticos, ideológicos, técnicos, teóricos, afectivos son cruciales, es que incorporamos una lectura de las políticas sociales a partir del concepto de dispositivo: esto supone desnaturalizar la concepción de las políticas como unidades homogéneas, compactas, que proceden de una unidad superior: el Estado (Dallorso, 2012).

Fernández (2009), retoma de Marí la idea de los dispositivos de poder en tanto redes de relaciones que se establecen entre elementos heterogéneos, discursivos y extradiscursivos, que articulan tres elementos básicos: la fuerza o la violencia (cómo elemento constitutivo del poder), el discurso del orden (el espacio de racionalidad o saber) y los imaginarios sociales.

Desde este punto el análisis incluye las siguientes dimensiones:

- La consideración de la inmanencia entre el poder-saber. Entre el saber y el poder no existe relación alguna de exterioridad, a su vez, todas las relaciones constituyen "focos locales" de poder-saber (Foucault, 2014). La noción de dispositivo propuesta por Foucault es una propuesta que incluye el análisis de las relaciones de poder y su relación entre lo discursivo y lo extradiscursivo.

- La indagación de las significaciones imaginarias sociales, los universos de significaciones imaginarias que instituyen y son instituidos en una sociedad forman parte de las luchas de poder, en tanto las sociedades participan en el plano simbólico del poder de conservar o transformar el mundo a partir de la transformación, invención o conservación de sus significaciones (Fernández, 2009). 
Cuando imperan las lógicas liberales, a la ausencia de recursos para la implementación de dispositivos que materialicen políticas públicas se suman acciones que por omisión producen espacios y poblaciones vulnerabilizadas: el creciente desempleo y precarización laboral, el considerable aumento en las tarifas de servicios básicos y elementales (tarifas de luz, gas, combustible y transporte), la falta de asistencia de salud en cercanía, la escasez de agua potable, la ausencia de recolección de residuos, son elementos que producen procesos de vulneraciones en las poblaciones que viven y trabajan en estas zonas de vulnerabilización, a partir de estrategias de poder biopolíticas (Foucault, 2014).

Pero, además, esta vulnerabilización, se enlaza con modos de producción de subjetividad. La disputa hegemónica la disputa sobre cuáles subjetividades promover y cuáles subjetividades desalentar es trasladada a los grupos. Se promueven de este modo sentidos y prácticas vinculadas a la competencia, el individualismo, el temor a la diferencia, la diferencia como amenaza, que impacta en fragmentaciones en el lazo social y en los vínculos comunitarios (Fernández, 2009).

La producción de subjetividad actual se da en un marco de condiciones sociohistóricas de corte neoliberal, donde las significaciones imaginarias sociales del éxito, el dominio y el instante operan como referentes y traman biografías caracterizadas por una plus-conformidad y la urgencia de satisfacción (Castoriadis, 2010).

Los anclajes que las instituciones debieran ofrecer tambalean, se insignifican, se vacían y, por lo tanto, desamparan. El resultado, entonces, es la constitución de sujetos para quienes no significan gran cosa los referentes de comunidad y pertenencia, el sentido de la propuesta, la proyección de un mundo distinto ni el cuestionamiento de su propio malestar y sufrimiento (Fernández, 2011).

Tomando los aportes de Foucault, Deleuze (1989), define a los dispositivos, como "máquinas de hacer ver y hacer oír" que poseen curvas de visibilidad y de enunciación, regímenes de saber y de poder. Las líneas que atraviesan a los dispositivos son líneas de fuerza que conectan distintos puntos (focos locales de ejercicio de poder y resistencias), pero también líneas de objetivación y de subjetivación. No es seguro que todos los dispositivos contengan este proceso de subjetivación que se sustrae de las relaciones de fuerzas establecidas. Los dispositivos contienen su aspecto histórico, las líneas del pasado reciente y las líneas del futuro próximo: la parte de lo actual y del acontecer. Pero el poder sólo es una dimensión estratificada del agenciamiento, este es la unidad mínima que produce los enunciados, que son siempre colectivos y que ponen en juego devenires, acontecimientos y multiplicidades, el diagrama o máquina abstracta tiene líneas de fuga que son, más que fenómenos de resistencia, potencias de desterritorialización y creación. Parafraseando a Guattari (1996), las transformaciones sociales pueden producirse a escala molecular, en dispositivos para cambiar los entornos, las instituciones y las comunidades. La posibilidad de constituir y visibilizar nuevos lenguajes es también la posibilidad de crear nuevos instituyentes, nuevas interpretaciones y comprensiones del mundo: 
Nuestra supervivencia en este planeta está amenazada no sólo por las degradaciones ambientales sino también por la degradación del tejido de solidaridades sociales y de los modos de vida psíquico que conviene, literalmente, reinventar (...) La única finalidad aceptable de las actividades humanas es la producción de una subjetividad que auto-enriquezca de manera continua su relación con el mundo (Guattari, 1996, pp.34-35).

Ana Fernández plantea que el debate dispositivo-agenciamiento queda abierto, por lo que propone pensar los dispositivos como creadores de condiciones de posibilidad para que se produzcan unos agenciamientos y no otros. Los agenciamientos ponen en común ciertas multiplicidades en las que los sujetos son pensados procesualmente, esto implica una relación transversal. Cuando se produce un agenciamiento, lo magmático hace forma y se produce un sentido (Fernández, 2009).

En esta línea, tomamos los aportes de Salazar Villalva (2003), para quién el campo de la investigación-intervención implica un desplazamiento de sentido desde el dispositivo como máquina social de control hacia la construcción intencional de máquinas para hacer ver y decir singulares "máquinas metodológicas" de reflexión, siempre abiertas, que producen visibilidad en los campos de la experiencia social y que se basan en la incertidumbre del investigador sobre el devenir del proceso investigativo y en la construcción de vínculos de reconocimiento del otro:

El dispositivo supone la creación colectiva de los saberes en cuestión y su acción es política, por cuanto introduce una iniciativa de reflexión posible para los actores sociales con quienes trabaja. No extrae información del campo, sino que introduce al investigador en un campo de reflexión compartida (Salazar Villalba, 2004, p. 297).

El término subjetividad nos conduce a pensar la articulación entre los modos sociales de sujeción y los restos no sujetados, las instancias de potencia que generan los colectivos, sus posibilidades de invención.

Insiste entonces la pregunta acerca del ejercicio de derechos y el derecho a la salud. ¿Es suficiente con la existencia de una legislación, o un establecimiento, servicio de salud para que pueda ejercerse el derecho a la salud? ¿Si la misma es concebida desde lógica de mercado y las personas concebidas como usuarios de un servicio, es suficiente pensar en términos de acceso a la institución de salud?

Proponemos entonces: Entender a las políticas públicas cómo dispositivos sociohistóricos, vinculados con las estrategias de poder que se efectúan a su vez a través de otros dispositivos agenciamientos, encuadres diseñados e implementados desde la localía, por los actores intervinientes en un determinado campo. Ya que si bien, los dispositivos socio históricos actuales producen zonas de vulnerabilización, fragmentaciones del lazo social y modos de subjetivación propios del individuo consumidor, que trocan derechos por mercancías, también es posible observar y 
promover, diferentes transformaciones y focos de resistencia que permiten localizar situaciones dónde se produce subjetividad.

Esto puede vincularse con el aumento de la participación de la comunidad en los procesos de toma de decisión y evaluación, la presencia de organización para la gestión de necesidades y recursos, la existencia y el reconocimiento de redes (formales e informales), el reconocimiento de referentes en su interior y la concepción de la política como herramienta de transformación social.

Se trata de que las prácticas de los equipos y los sectores de la población a los cuales las políticas públicas se orientan tengan el efectivo reconocimiento de ser sujetos de derecho, desde un aspecto relacional al reconocerse y ser reconocidos como protagonistas participantes del proceso.

\section{Caracterización del territorio}

El barrio está ubicado en la periferia de la ciudad y ha tenido un crecimiento importante en los últimos 18 años. Este crecimiento poblacional ha llevado a la convivencia de grupos de gran variación socioeconómica. Se encuentra localizado muy cerca de dos caminos de acceso interurbano, en proximidad a medios de transporte públicos (colectivos).

El tipo de viviendas es precario, los principales materiales son maderas y chapas. Las calles son de tierra, sin asfalto y los días de lluvia se tornan intransitables. No se cuenta con servicio de recolección de residuos, es posible observar basurales en esquinas y puertas de domicilios.

El territorio cuenta con la cercanía de una escuela primaria pública a la que asisten gran parte de los niños de la zona. Este espacio no cuenta con instituciones para el abordaje de la salud, ni públicas ni privadas.

En muchos casos los vecinos asisten a servicios de salud ubicados en el centro de la ciudad. Generalmente esto implica el traslado en transporte público, hacia los hospitales provinciales que se encuentran en la ciudad (Se asiste en transporte público, es necesario contar con el dinero para abonar el boleto para los integrantes, las distancias suelen ser de varios kilómetros, a razón que no hay una línea que conecte de manera directa el barrio con el centro urbano).

Los recursos en territorio para las franjas etarias hasta los 18 años en salud mental están ausentes. Su alta demanda en atención es derivada a centros urbanos centralizados. No se cuenta con espacios de esparcimiento y recreación.

A medida que realizábamos recorridas por el barrio y efectuábamos entrevistas informales, vecinos fueron enunciando variadas problemáticas: violencia hacia la mujer, abuso sexual y maltrato infantil. Temor e inseguridad por la formación de bandas de adolescentes. Preocupación e intranquilidad de parte de los adultos por dejar a los niños solos cuando salen a trabajar, por los múltiples riesgos que puedan correr y la necesidad de encontrar condiciones seguras para sus cuidados. 
Entrelas principalesproblemáticasidentificadas, se señala un aumento del diagnóstico de desnutrición y de enfermedades respiratorias, entre ellas bronquiolitis. En relación a las problemáticas señaladas por los participantes: violencia intrafamiliar, abuso sexual infantil, embarazos adolescentes no intencionales, consumo problemático de sustancias y adicciones.

Otra cuestión que emerge con regularidad en el diálogo con los vecinos es que "el barrio está asolado por robos, hurtos y la comercialización de drogas". Los jóvenes son señalados como peligrosos, responsables de los delitos y conflictos, se vincula esto al supuesto consumo de sustancias estimulantes.

En el barrio que hemos caracterizado se observa que no hay espacios de atención de la salud en el territorio, entonces ¿de qué manera los efectores de salud pueden desde sus acciones promover salud mental y ejercicio de derecho a la salud? No intervenir en estas situaciones hace que la acción por omisión siga promoviendo modos de subjetivación que entiendan la salud como un objeto de consumo, una mercancía, en lugar de producir las lógicas colectivas que permitan subjetivarla como un derecho para ser reclamado y exigido. A su vez, que estos puedan constituirse también en actores partícipes de las decisiones de gestión y elaboración de las políticas, a partir de una coordinación estatal.

A continuación, presentamos la modalidad de intervención que el equipo de salud, a partir del proyecto de extensión elaboró para este territorio, sobre la misma se produce un dispositivo, que desde lo micro, dispone a movilizar focos de poder cristalizados y que contribuye a modos de producción de subjetividad en los que se apuesta a resistir a modos liberales de concebir los lazos sociales y la salud.

\section{El barrio de los chicos}

Ante la ausencia de un centro de salud en territorio, como equipo extensionista de salud planteamos un dispositivo de intervención de carácter territorial con base en la comunidad y con un componente de Atención Primaria de la Salud y promoción de la salud, de acuerdo con los lineamientos de las políticas públicas desde un marco de derechos.

Los vecinos nos ofrecieron un espacio físico que funcionaba como comedor y escuelita de apoyo para montar el espacio de salud algunos días a la semana.

En un primer momento realizamos unas primeras entrevistas, en las que se fueron enunciando distintas situaciones de violencias: de género, intrafamiliares, entre vecinos. Se presentaba la fragmentación de los lazos que se reflejaba en barreras imaginarias en el territorio y los enfrentamientos entre diferentes grupos, y se verbalizaban diferencias dentro del barrio de acuerdo a la procedencia de los habitantes, migrantes, inmigrantes, "el barrio de los paraguayos", "el barrio de los bolivianos".

El primer día de trabajo en el barrio, recorrimos las calles con un grupo de chicos que 
participarían del taller, mientras nos iban diciendo dónde estaban sus conocidos, a quienes debíamos invitar a los talleres y quienes eran chicos con los que compartían cosas en la escuela o en la calle. Nos señalaron lugares a los que no deberíamos ir porque estaban "los cirujas". En esa ocasión cuando uno de los niños no podía concurrir al taller porque debía ir a trabajar con sus padres, una de las nenas, en un gesto de desprecio dijo: "A estos déjalos, si no parecen niños, parecen cartoneros".

Para empezar, desde el equipo se elaboró un cronograma de atención a la salud: odontología, clínica médica, jornadas de vacunación y salud mental. Uno de los pedidos que insistía era que éste psicología. A partir de estos intercambios, desde el espacio de salud mental se propuso un espacio destinado a infancias y juventudes, en principio pensado para la promoción de derechos en un sentido amplio.

La propuesta tuvo diferentes momentos, estos fueron construyéndose colectivamente. En los primeros encuentros fue recreativa, lúdica.

Se propuso generar un espacio estilo feria, de juegos colaborativos y en posta, que convoque. Estos primeros encuentros rápidamente nos expusieron las limitaciones de la propuesta planteada. Los primeros encuentros tenían cómo característica principal la dispersión, la agresión. No poder jugar, era no contar con un espacio de pausa, de elaboración de lo acontecido a partir de elementos simbólicos y creativos. $\mathrm{El}$ juego permite desplegar otros mundos posibles, anticipar, resignificar. El primer paso, entonces, era poder generar las condiciones para jugar.

Los jóvenes contaban sentirse excluidos y señalados, temidos por vecinos de barrios cercanos. Las familias planteaban que varios niños y niñas, se encontraban medicalizados y en tratamiento psicológico, estos eran demandados a partir de observar reiterados "problemas de conducta", agresiones entre compañeros de curso.

El desafío consistió generar espacios que capaces de producir otros modos de ser y estar, que conmueven estos etiquetamientos y permitan su deconstrucción. ¿Es posible conmover las representaciones de peligrosidad que estigmatizan estas vidas?

El segundo momento de las intervenciones consistió entonces en construir un espacio lúdico, mediado por el dibujo. Este tiempo fue largo, a partir de estos encuentros las coordinadoras pudieron ir acompañando diferentes circunstancias de salud que se presentaban a medida que la permanencia posibilitaba el reconocimiento y un mayor diálogo con los niños y sus familias. Aquí se trabajaron diferentes acompañamientos y también la construcción de espacios singulares que en muchos casos incluían entrevistas con el consultorio de psicología, consultorio físicamente improvisado que funcionaba en la cocinita del espacio.

Comenzó a interrogarnos la manera de conmover la propuesta para que entre los niños y niñas que participaban pudieran realizar juegos en grupo. Generar espacios que posibiliten momentos de escucha y de protagonismo de las voces de participantes, en que las producciones que realizaran pudieran ser construidas a partir de sus testimonios. A su vez, propiciar que a partir de la tarea pudieran establecerse nuevas modalidades en los vínculos, con el grupo y con la comunidad, 
que permitieran también nuevos existenciarios en el marco de la promoción y la protección de los derechos de los niños, niñas y adolescentes.

Comenzamos con la lectura de cuentos. En un primer momento libros ya editados y publicados. Poco a poco, los participantes del taller comenzaron a hacer propuestas. En conjunto propusieron comenzar a escribir una historia entre todos, así el taller de recreación pasó a ser el taller de dibujos, y este a ser el "taller de títeres". Construir la historia implicó pensar los personajes, sus características, modos de relacionarse, su vestuario y el armado de las escenas, para poder presentarla a fin de año en una muestra abierta en la calle.

Observamos a partir de la construcción de personajes como se modificaron algunos de los "lugares comunes" vinculados a la estigmatización: en este juego, quien era nombrado como "niño violento, niño problema" elaboró un nuevo personaje junto a sus compañeros, dónde pasó de ser "el más malo de todos" a un niño salvador que protegía a los demás de diferentes peligros.

El espacio de niñez comenzó de a poco a tener una continuidad argumental entre los encuentros, acompañados de las intervenciones de las talleristas, los niños comenzaron a reglar sus juegos, a construir sus propias reglas y acuerdos: construyeron un "contrato" del taller dónde no podían pelearse.

Las situaciones que atraviesan quienes participan del taller son complejas y deben contar con un abordaje integral, que no puede ser resuelto ni desde un único sector, ni disciplina. Es imprescindible establecer redes, diálogos intersaberes y realizar una intervención integral sobre los diferentes determinantes, una apuesta permitió de algún modo dar lugar a la producción de nuevos lazos y formas de comunicarse y vincularse entre pares, con adultos, con el barrio y escuela.

Para el cierre del año, se realizó una presentación abierta de todos los trabajos que realizaron, esta jornada fue organizada en conjunto, cada niño y niña contaba que sabía hacer para enseñarle al grupo y poder hacer entre todos: guirnaldas, galletitas, cartelería, fueron algunas de las actividades. Finalmente, eligieron un nombre para el evento: "el barrio de los chicos", con ese lema, realizamos la recorrida en grupo por todas las casas invitando a las familias.

Ahora bien, ¿por qué situamos esta experiencia a partir de los desarrollos planteados en la primera etapa de este escrito? Ulloa (2011) sostiene la noción de salud mental, en el marco de la categoría de contrapoder, definida en términos de poder hacer mientras tanto. De este modo, un espacio de salud implica necesariamente el ejercicio de derechos, y el ejercicio de estos es producción de salud. El autor sitúa la política como un accionar sobre acciones, que vale también para todo accionar clínico, dónde los colectivos disponen de una capacidad inagotable de invención para promover una valentía suficiente para interrumpir la subjetividad serializada, mortecina y crear condiciones para la producción de procesos de subjetivación: 
"Entiendo que la movilidad política, fuera de los ámbitos clínicos, es imprescindible para inscribir la salud mental en términos de contrapoder, es decir, en términos de importante variable política, ya que en ese registro la salud mental coincide con una comunidad organizada en serio de forma democrática" (Ulloa, 2011 pp. 121).

Más que disponer de un espacio y tiempo para una consulta, es necesaria la lectura situada de cada grupo y comunidad particular, y analizar la potencialidad de cada dispositivo de producir subjetividades en el marco de derechos, cada vez.

\section{A modo de conclusión}

Poder intervenir, en este caso a partir del trabajo de investigación-acción, implica una apuesta por un mayor grado de visibilidad y enunciación que permita la producción de nuevos saberes sobre la realidad que se interroga. Al ser las relaciones de saber y poder inmanentes, se producen aperturas y movimientos en los focos locales de ejercicio de poder y resistencias capaces de abrir otros mundos y devenires posibles. Es por esto, que sostenemos que el análisis de las políticas públicas debe introducir la lectura de los dispositivos de atención y de las subjetividades que producen.

Dado que las normativas establecen el derecho a la salud como un derecho universal, para todos y todas los/as habitantes del territorio argentino, nos interrogamos si los dispositivos de salud son productores de la salud como derecho. Consideramos la importancia de delinear la categoría de accesibilidad: de acuerdo con cómo sea pensada e implementada, ella estará alineada o bien con la lógica de servicio, o bien con la lógica de derecho. En términos de la investigación aquí presentada: que las políticas públicas dispongan entre sus elementos normativos a 'sujetos de derechos' ¿garantiza su efectiva producción?

El artículo delimita las relaciones de inmanencia existentes entre las políticas públicas, los dispositivos concretos que los materializan y los tipos de subjetividad. Es decir, si bien los Estados liberales producen unos tipos de políticas sociales en lógica de servicio y de usuarios en tanto consumidores de tales servicios, es posible generar otro tipo de respuestas por parte de los efectores que genere intervenciones que desde lo situacional sean en el marco de ejercicio de derechos.

Más que analizar la accesibilidad, se vuelve clave y transversal relevar los elementos que se disponen en los dispositivos que implementan los efectores de políticas públicas para evaluar su potencialidad de producir la salud como derecho de los habitantes. Si así lo hacen, ello implicaría focos de resistencia a las lógicas neoliberales que producen la salud como objeto de consumo.

Se trata de generar pequeñas aperturas en la trama que, como rendijas de luz, posibiliten una problematización sobre enunciados, representaciones, sentidos $\mathrm{y}$ afectos instituidos para que aquello que se presenta como impuesto, externo y heterónomo pueda ser cuestionado y dimensionado como parte del campo de 
subjetivación para, de este modo, producir efectos subjetivos colectivos. Así, el derecho no es algo exterior que se obtiene o a lo que se accede, sino un elemento más que, en el juego de fuerzas de poder puede, junto con otros elementos, producir efectos subjetivos de salud.

La perspectiva desde la cual realizamos el trabajo tiene en cuenta la relación entre las instituciones y los modos de producción de subjetividad que generan las políticas públicas sociales.

Proponemos continuar, las intervenciones en territorio, desnaturalizar, visibilizar y problematizar los sentidos socialmente construidos (que contribuyen a la construcción de las zonas de vulnerabilización sobre ciertos grupos) a partir de propuestas que apunten a la construcción de lo común, a alojar a lo diferente y que contemplen la posibilidad de contener a la diversidad sin desigualar.

Reforzamos la importancia de que estas acciones no queden en experiencias aisladas y singulares, como casos excepcionales, sino que sean incorporadas y coordinadas desde las políticas públicas, a partir de un Estado presente que trabaje de manera organizada y en red con los diferentes actores involucrados. Para finalizar, señalamos la importancia de incorporar en todos los diferentes momentos de las políticas públicas (diseño, implementación, evaluación), la voz de los efectores de salud y de los principales destinatarios, priorizando la palabra de quienes habitan en las comunidades o territorios, de los trabajadores y la inclusión de sus perspectivas, necesidades, preocupaciones, redes de cuidado y modos de respuesta a las principales problemáticas que se presentan.

\section{Agradecimientos}

A Edith Alba Pérez, una imprescindible que vivió siempre comprometida con su tiempo.

\section{Notas}

\section{Datos de los proyectos}

Entre juguetes y cartones. Hacia la constitución de lazos comunitarios. Directora: Pérez, Edith. Coordinadora: Tomaino, Sandra. Facultad Psicología. Centro Universitario de Extensión. Facultad de Psicología, UNLP. (Res. Nº133/12).

Ejercicio de Derechos y Producciones de Subjetividad. Un estudio en redes intersectoriales en instituciones de La Plata y Gran La Plata. Segunda Parte. (I+D). Director: Juan Carlos Domínguez Lostalo. Codirección: María Antonia Luis. Secretaria de Ciencia y Técnica, Universidad Nacional de La Plata (SO56).

Ambos proyectos han sido evaluados por el comité de ética correspondiente, de este modo, hemos solicitado y obtenido el consentimiento informado de parte de todos/ as los involucrados/as en el proceso de intervención e investigación. 


\section{Referencias}

Abramovich, V. (2006). Una aproximación al enfoque de derechos en las estrategias y políticas de desarrollo. Revista de la CEPAL, 88, 33-50. HTTP://DX.DOI.ORG/10.18356/A48F3CCA-ES

Aguilo, J. C. (2005). Políticas sociales en Argentina: de la Sociedad de Beneficencia a la focalización compulsiva. The University of Texas at Austin. Teresa Lozano Long Institute of Latin American Studies.

Calmels, J. (2015). Experiencias en salud mental $y$ derechos humanos: aportes desde la política pública. Ministerio de Justicia y Derechos Humanos de la Nación. Secretaría de Derechos Humanos.

Carrizo Villalobos, C. (2016). Accesibilidad a los servicios de salud mental con perspectiva de diversidad de género. En el primer y segundo nivel de atención en la localidad de Alta Gracia, año 2014. Revista de Salud Pública, 20(3), 5970. HTTP://DX.DOI.ORG/10.31052/1853.1180.V20. N3.14424

Castoriadis, C. (2010/1983). La institución imaginaria de la sociedad (A. Vicens y M-A. Galmarini, Trads.). Tusquest.

Comes, Y. y Stolkiner, A. (2005). Si pudiera pagaría. Estudio sobre la accesibilidad simbólica de las mujeres usuarias pobres del AMBA a los servicios asistenciales estatales. Anuario de Investigaciones, 12, 137-143.

Comes, Y. y Stolkiner, A. (2007). Representaciones de derecho a la atención de la salud en un grupo de mujeres seleccionado. En Memorias XIV Jornadas de Investigación y Tercer Encuentro de Investigadores en Psicología del Mercosur. Buenos Aires: Facultad de Psicología - Universidad de Buenos Aires.

Comes, Y., Solitario, R., Garbus, P., Mauro, M., Czerniecki, S. y Vázquez, A. (2007). El concepto de accesibilidad: perspectiva relacional entre población y servicios. Anuario de Investigaciones, 14, 201-209.

D’Agostino, A, Vidal, I. y Veloz, J. (2018). Psicología institucional. Un campo de problemas. Edulp.

Dallorso, N. S. (2012). Notas sobre el uso del concepto de dispositivo para el análisis de programas sociales. Espiral, Estudios sobre Estado y Sociedad, 19(54), 43-74.
Danani, C. (2012). Procesos de reformas y configuración de un nuevo régimen de política social: el trabajo, la seguridad social y los planes sociales en Argentina. La otra década de reformas de las políticas sociales y laborales. Argentina, 2002-2010. Revista de Ciencias Sociales, 135-136, 59-72.

Del Cueto, A. M. (2014). Salud mental comunitaria. Vivir, pensar, desear. FCE.

Deleuze, G. (1989). ¿Qué es un dispositivo? En VV.AA., Michel Foucault, filósofo (pp. 155-163). Gedisa.

Domínguez Lostaló, J. C. (1996). ¿Es necesario encerrar? El derecho a vivir en comunidad. Cuadernos de Caleuche.

Fernández, A.M. (2008). Políticas de investigación e investigación de las políticas. En A. Stolkiner (Comp.) Las dimensiones politicas de la investigación (pp. 25-34). JVE.

Fernández, A.M. (2009). Las lógicas colectivas. EUDEBA.

Fernández, A.M. (2011). Política y subjetividad. Biblos.

Foucault, M. (2014). El poder, una bestia magnifica. Sobre el poder, la prisión y la vida. Siglo XXI.

Fleury, S. (2002). Políticas sociales y ciudadanía. Umbrales. Revista del Postgrado en Ciencias del Desarrollo, 11, 189-218.

Guattari, F. (1996). Acerca de la producción de subjetividad. Caosmosis. Manantial.

Landini, F, González Cowes, V. y D’Amore, E. (2014). Hacia un marco conceptual para repensar la accesibilidad cultural. Cadernos Saúde Pública, 30(2), 231-244. http://dx.doi.org/10.1590/0102$311 \times 00030313$

Pautassi, L. (2010). El aporte del enfoque de derechos a las políticas sociales. Una breve revisión. Taller de expertos. Protección social, pobreza y enfoque de derechos: vínculos y tensiones. Chile: ONU/ CEPAL.

Pérez, E. A. (2014). Psicología institucional. EDULP.

Salazar Villalva, C. M. (2003). Dispositivos: máquinas de visibilidad. Anuario de Investigaciones UAM-X, 3, 291-299. 
Solitario, R; Garbus, P. y Stolkiner, A. (2008). Derechos, ciudadanía y articulación en salud: su relación con la accesibilidad simbólica a los servicios. Anuario de Investigaciones, 15, 263269.

Stolkiner, A. (2008). Las dimensiones políticas de la investigación. JVediciones.

Stolkiner, A. (2015). Derechos humanos y salud desde el pensamiento médico social /salud colectiva latinoamericano. En Llambias Wolff (Ed.), La enfermedad de los sistemas de salud. Miradas críticas y alternativas (pp. 136-150). York University.
Stolkiner, A. (2017). Asistir e investigar: el proceso de investigación en el campo de la salud mental. Clepios. Revista de Profesionales en Formación en Salud Mental, 3(22), 124-130.

Ulloa, F. (2011). La salud ele-mental. Libros del Zorzal. 